
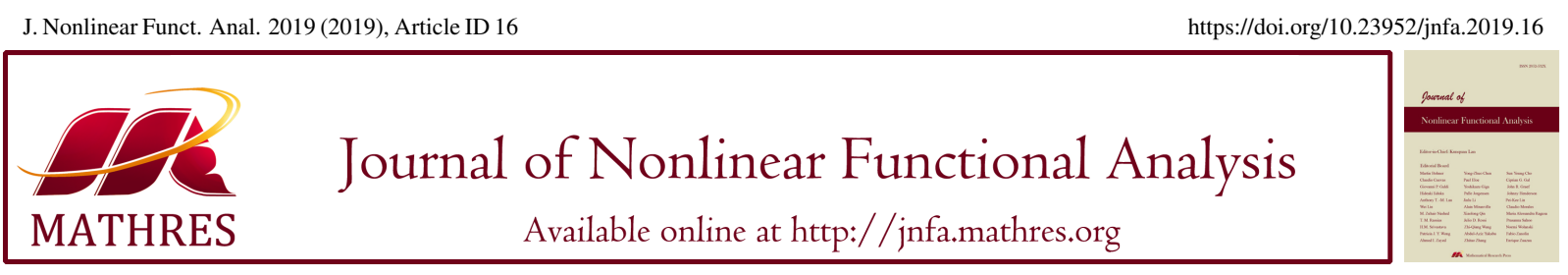

\title{
ON A THREE-POINT FRACTIONAL INTEGRAL BOUNDARY VALUE PROBLEM ON THE HALF-LINE
}

\author{
ABDELLATIF GHENDIR AOUN* \\ Department of Mathematics, Faculty of Exact Sciences, Hamma Lakhdar University, 39000 El-Oued, Algeria \\ Laboratory "Theory of Fixed Point and Applications", ENS, BP 92 Kouba. Algiers, 16006, Algeria
}

\begin{abstract}
In this paper, we are concerned with the existence of solutions to fractional differential equations subject to RiemannLiouville fractional integral boundary conditions. By means of a recent fixed point theorem, sufficient conditions are obtained that guarantee the existence of at least one solution. Two examples illustrate the applicability of our main result.
\end{abstract}

Keywords. Boundary value problem; Infinite interval; Fractional differential equation; Fixed point theorem.

2010 Mathematics Subject Classification. 34A08, 34B40.

\section{INTRODUCTION}

In this paper, we consider the following boundary value problem (BVP)

$$
\left\{\begin{array}{l}
D_{0^{+}}^{\alpha} u(t)+f\left(t, u(t), D_{0^{+}}^{\alpha-1} u(t)\right)=0, \quad t \in(0,+\infty), \\
u(0)=0, \quad \lim _{t \rightarrow+\infty} D_{0^{+}}^{\alpha-1} u(t)=\beta I_{0^{+}}^{\alpha-1} u(\eta),
\end{array}\right.
$$

where $1<\alpha \leqslant 2, \eta>0$ and $\beta>0$ satisfy $0<\beta \eta^{2 \alpha-2}<\Gamma(2 \alpha-1), D_{0^{+}}^{\alpha}$ is the standard RiemannLiouville fractional derivative and $I_{0^{+}}^{\alpha-1}$ is the standard Riemann-Liouville fractional integral.

Fractional equations are natural generalizations of the classical integer-order differential equations. They turn out to be useful for modeling dynamics of many processes involving complex systems that can found in science and engineering. For instance, integral boundary conditions appear in blood flow models as well as in chemical engineering. Fractional integral conditions may provide good information rather than classical local boundary conditions.

Recently, many results have been concerned with fractional differential equations and great progress has been made in the study of BVPs involving differential equations; see, e.g., [1]-[14] and references therein. Guezane-Lakoud, Khaldi [6] studied the following boundary value problem with fractional

E-mail address: ghendirmaths@gmail.com.

Received January 1, 2019; Accepted April 14, 2019.

(C)2019 Journal of Nonlinear Functional Analysis 
integral boundary conditions in bounded interval

$$
\left\{\begin{array}{l}
{ }^{c} D_{0^{+}}^{q} x(t)+f\left(t, x(t),{ }^{c} D_{0^{+}}^{p} x(t)\right)=0, \quad 0<t<1,1<q \leqslant 2,0<p<1 \\
x(0)=0, \quad x^{\prime}(1)=\alpha I_{0^{+}}^{p} x(1),
\end{array}\right.
$$

where ${ }^{c} D^{q}$ denotes the Caputo fractional derivative.

In [9], Shen, Zhou, and Yang established existence of positive solutions for the following BVP

$$
\left\{\begin{array}{l}
D_{0^{+}}^{\alpha} u(t)+f\left(t, u(t), D_{0^{+}}^{\alpha-1} u(t)\right)=0, \quad t \in(0,+\infty), \\
u(0)=0, \quad u^{\prime}(0)=0, \quad D_{0^{+}}^{\alpha-1} u(+\infty)=\sum_{i=1}^{m-2} \beta_{i} u\left(\xi_{i}\right),
\end{array}\right.
$$

where $2<\alpha \leqslant 3$. Using the Schauder fixed point theorem, they showed the existence of one solution under suitable growth conditions imposed on the nonlinear term. Su and Zhang [10] discussed the existence of unbounded solutions and used the Schauder's fixed point theorem to prove the existence of solutions for the following BVP

$$
\left\{\begin{array}{l}
D_{0^{+}}^{\alpha} u(t)+f\left(t, u(t), D_{0^{+}}^{\alpha-1} u(t)\right)=0, \quad t \in(0,+\infty), \\
u(0)=0, \quad u^{\prime}(0)=0, \quad D_{0^{+}}^{\alpha-1} u(\infty)=u_{\infty}, \quad u_{\infty} \in \mathbb{R},
\end{array}\right.
$$

where $1<\alpha \leqslant 2$. Yu, Wang, and Guo [13] considered the solvability for the following integral boundary value problem of fractional differential equation:

$$
\left\{\begin{array}{l}
D_{0^{+}}^{\alpha} u(t)+f\left(t, u(t), D_{0^{+}}^{\alpha-1} u(t)\right)=0, \quad t \in(0,+\infty), \\
u(0)=0, \quad D_{0^{+}}^{\alpha-1} u(\infty)=\int_{\eta}^{+\infty} g(t) u(t) d t,
\end{array}\right.
$$

where $1<\alpha \leqslant 2, f \in C([0,+\infty) \times \mathbb{R} \times \mathbb{R}, \mathbb{R}), \eta \geqslant 0, g(t) \in L^{1}[0,+\infty)$ and $\int_{\eta}^{+\infty} g(t) u(t) d t<\Gamma(\alpha)$.

The result presented in this paper is a continuation of previous works and is concerned with a boundary value problem of fractional order sets on the half-axis. Our main result is mainly motivated by [6], [9], [10], [13]. To overcome the compactness difficulty of fixed point operators, a special Banach space is considered. Our results allow the integral condition to depend on the fractional integral $I_{0^{+}}^{\alpha-1} u$, which leads to additional difficulties. The organization of the paper is as follows. In Section 2, some definitions and lemmas, which are crucial to our discussion are presented. Related lemmas, which are necessary to the fixed point formulation and the main existence theorem of solutions are given in Section 3. We end this paper by presenting two examples to illustrate our theoretical result.

\section{PRELIMINARIES}

We first collect some definitions and lemmas on the fractional calculus (see [15] and [16] for more details). One of the basic tools for the fractional calculus is the Gamma function which extends the factorial to positive real numbers (and even to complex numbers with positive real parts).

Definition 2.1. For $\alpha>0$, the Euler gamma function is defined by

$$
\Gamma(\alpha)=\int_{0}^{+\infty} t^{\alpha-1} e^{-t} d t
$$

Definition 2.2. Let $p>0, q>0$, the Euler beta function is defined by

$$
B(p, q)=\int_{0}^{1} t^{p-1}(1-t)^{q-1} d t
$$


Proposition 2.3. Let $\alpha>0, p>0, q>0$ and $n$ a positive integer. Then

$$
\Gamma(\alpha+1)=\alpha \Gamma(\alpha), \quad \Gamma\left(n+\frac{1}{2}\right)=\frac{\sqrt{\pi} \Gamma(2 n+1)}{2^{2 n} \Gamma(n+1)}, \quad B(p, q)=\frac{\Gamma(p) \Gamma(q)}{\Gamma(p+q)},
$$

and

$$
\Gamma(\alpha+n)=\alpha(\alpha+1)(\alpha+2) \ldots(\alpha+n-1) \Gamma(\alpha) .
$$

In particular,

$$
\begin{aligned}
& \Gamma(1)=\int_{0}^{+\infty} e^{-t} d t=1, \quad \Gamma\left(\frac{1}{2}\right)=\sqrt{\pi}, \\
& \Gamma(n+1)=n !, \quad \Gamma\left(n+\frac{1}{2}\right)=\frac{\sqrt{\pi}(2 n) !}{2^{2 n} n !} .
\end{aligned}
$$

Definition 2.4. The fractional integral of order $\alpha>0$ for function $h$ is defined as

$$
I_{0^{+}}^{\alpha} h(t)=\frac{1}{\Gamma(\alpha)} \int_{0}^{t}(t-s)^{\alpha-1} h(s) d s
$$

provided the right side is point-wise defined on $(0,+\infty)$.

Definition 2.5. For a function $h$ given on the interval $[0,+\infty)$, the Riemann-Liouville fractional derivative of order $\alpha>0$ of $h$ is defined by

$$
D_{0^{+}}^{\alpha} h(t)=\left(\frac{d}{d t}\right)^{n} I_{0^{+}}^{n-\alpha} h(t)=\frac{1}{\Gamma(n-\alpha)}\left(\frac{d}{d t}\right)^{n} \int_{0}^{t} \frac{h(s)}{(t-s)^{\alpha-n+1}} d s,
$$

where $n=[\alpha]+1$.

Lemma 2.6. ([16]) Let $\alpha>0$. Then

$$
I_{0^{+}}^{\alpha} D_{0^{+}}^{\alpha} u(t)=u(t)+c_{1} t^{\alpha-1}+c_{2} t^{\alpha-2}+\ldots+c_{n} t^{\alpha-n}
$$

for some $c_{i} \in \mathbb{R}, i=1,2, \ldots, n, n=[\alpha]+1$.

Proposition 2.7. In this paper, we need the following composition relations:

(a) $D_{0^{+}}^{\alpha} I_{0^{+}}^{\alpha} h(t)=h(t), \quad \alpha>0, \quad h(t) \in L^{1}[0,+\infty)$.

(b) $D_{0^{+}}^{\alpha} I_{0^{+}}^{\gamma} h(t)=I_{0^{+}}^{\gamma-\alpha} h(t), \quad \gamma>\alpha>0, \quad h(t) \in L^{1}[0,+\infty)$.

(c) $I_{0^{+}}^{\alpha} I_{0^{+}}^{\gamma} h(t)=I_{0^{+}}^{\alpha+\gamma} h(t), \quad \alpha>0, \quad \gamma>0 \quad h(t) \in L^{1}[0,+\infty)$.

(d) $D_{0^{+}}^{\alpha} t^{\lambda}=\frac{\Gamma(\lambda+1)}{\Gamma(\lambda-\alpha+1)} t^{\lambda-\alpha}$, for $\lambda>-1$, giving in particular $D_{0^{+}}^{\alpha} t^{\alpha-m}=0$, $m=1,2, \ldots, N$, where $N$ is the smallest integer greater than or equal to $\alpha$.

(e) $I_{0^{+}}^{\alpha} t^{\lambda}=\frac{\Gamma(\lambda+1)}{\Gamma(\alpha+\lambda+1)} t^{\alpha+\lambda}, \quad \alpha>0, \quad \lambda>-1$.

(f) (Properties 1.3, of the Gamma function, in [15]) if $1 \leqslant \alpha \leqslant 2$ then $\frac{1}{\Gamma(\alpha)} \geqslant 1$.

The following result is needed to prove our main existence result. This is a nonlinear alternative for the Krasnosel'skii' s fixed point theorem [17].

Theorem 2.8. [17] Let $U$ be an open set in a closed, convex set $C$ of a Banach space E. Assume that $0 \in U, F(\bar{U})$ bounded and $F: \bar{U} \rightarrow C$ is given by $F=F_{1}+F_{2}$, where $F_{1}: \bar{U} \rightarrow E$ is continuous and completely continuous and $F_{2}: \bar{U} \rightarrow E$ is a contraction, i.e., there exists a constant $0<l<1$ such that $\left\|F_{2}(x)-F_{2}(y)\right\| \leqslant l\|x-y\|$, for all $x, y \in \bar{U}$. Then either,

(A1) $F$ has a fixed point in $\bar{U}$, or

(A2) There is a point $u \in \partial U$ and $\lambda \in(0,1)$ with $u=\lambda F(u)$. 


\section{RELATED LEMmAS AND THE EXISTENCE THEOREM}

Define the spaces

$$
X=\left\{u \in C([0,+\infty), \mathbb{R}): \quad \sup _{t \geq 0} \frac{|u(t)|}{1+t^{\alpha}}<+\infty\right\}
$$

with the norm

$$
\|u\|_{X}=\sup _{t \geq 0} \frac{|u(t)|}{1+t^{\alpha}}
$$

and

$$
Y=\left\{u \in X, \quad D_{0^{+}}^{\alpha-1} u \text { exists, } \quad D_{0^{+}}^{\alpha-1} u \in C([0,+\infty), \mathbb{R}), \quad \sup _{t \geq 0}\left|D_{0^{+}}^{\alpha-1} u(t)\right|<+\infty\right\}
$$

with the norm

$$
\|u\|_{Y}=\max \left\{\sup _{t \geq 0} \frac{|u(t)|}{1+t^{\alpha}}, \quad \sup _{t \geq 0}\left|D_{0^{+}}^{\alpha-1} u(t)\right|\right\} .
$$

Lemma 3.1. $\left(X,\|\cdot\|_{X}\right)$ and $\left(Y,\|\cdot\|_{Y}\right)$ are Banach spaces.

Proof. Let $\left\{u_{n}\right\}_{n \in \mathbb{N}}$ be a Cauchy sequence in the space $\left(X,\|\cdot\|_{X}\right)$. Then

$$
\forall \varepsilon>0, \exists N>0 \text { such that }\left\|u_{n}-u_{m}\right\|_{X}<\varepsilon \text { for any } n, m>N,
$$

i.e.

$$
\forall \varepsilon>0, \exists N>0 \text { such that }\left|\frac{u_{n}(t)}{1+t^{\alpha}}-\frac{u_{m}(t)}{1+t^{\alpha}}\right|<\varepsilon \text { for any } t \in[0,+\infty) \text { and } n, m>N .
$$

Thus $\left\{\frac{u_{n}(t)}{1+t^{\alpha}}\right\}_{n \in \mathbb{N}}$ for $t \in[0,+\infty)$ is a Cauchy sequence in $\mathbb{R}$, too. So, there exists a $l(t) \in \mathbb{R}$ such that $\lim _{n \rightarrow+\infty}\left|\frac{u_{n}(t)}{1+t^{\alpha}}-l(t)\right|=0, t \in[0,+\infty)$. This implies $\lim _{n \rightarrow+\infty}\left\|u_{n}-u\right\|_{X}=0$, where $u(t)=l(t)\left(1+t^{\alpha}\right)$. So $\left(X,\|\cdot\|_{X}\right)$ is a Banach space. In a similar way, we can prove that $\left(Y,\|\cdot\|_{Y}\right)$ is a Banach space. Let $\left\{u_{n}\right\}_{n \in \mathbb{N}}$ be a Cauchy sequence in $\left(Y,\|\cdot\|_{Y}\right)$. Then $\left\{u_{n}\right\}_{n \in \mathbb{N}}$ is also a Cauchy sequence in space $\left(X,\|\cdot\|_{X}\right)$. Thus, $\lim _{n \rightarrow+\infty} \frac{u_{n}(t)}{1+t^{\alpha}}=\frac{u(t)}{1+t^{\alpha}}$ for some $u \in X$, i.e.

$$
\forall \varepsilon>0, \exists N>0 \text { such that }\left|\frac{u_{n}(t)}{1+t^{\alpha}}-\frac{u(t)}{1+t^{\alpha}}\right|<\varepsilon \text { for any } t \in[0,+\infty) \text { and } n>N .
$$

Then there exists a positive constant $M>0$ such that $\frac{\left|u_{n}(t)\right|}{1+t^{\alpha}} \leqslant M, n \in \mathbb{N}$. We claim that $\left\{D_{0^{+}}^{\alpha-1} u_{n}(t)\right\}_{n \in \mathbb{N}}$ converges. In fact, if $\alpha=2$, then

$$
\lim _{n \rightarrow+\infty} u_{n}^{\prime}(t)=\frac{d}{d t}\left(\lim _{n \rightarrow+\infty} u_{n}(t)\right)=u^{\prime}(t)
$$

In the case $1<\alpha<2$, we get

$$
\lim _{n \rightarrow+\infty} D_{0^{+}}^{\alpha-1} u_{n}(t)=\frac{1}{\Gamma(2-\alpha)} \lim _{n \rightarrow+\infty} \frac{d}{d t} \int_{0}^{t}(t-s)^{1-\alpha}\left(1+s^{\alpha}\right) \frac{u_{n}(s)}{1+s^{\alpha}} d s .
$$

Moreover, for any $t \in[0,+\infty)$ and $1<\alpha<2$, we get

$$
\left|\int_{0}^{t}(t-s)^{1-\alpha}\left(1+s^{\alpha}\right) \frac{u_{n}(s)}{1+s^{\alpha}} d s\right| \leqslant M \int_{0}^{t}(t-s)^{1-\alpha}\left(1+s^{\alpha}\right) d s
$$


Since

$$
\begin{aligned}
\int_{0}^{t}(t-s)^{1-\alpha}\left(1+s^{\alpha}\right) d s & =\int_{0}^{1} t^{1-\alpha}(1-\tau)^{1-\alpha}\left(1+t^{\alpha} \tau^{\alpha}\right) t d \tau \\
& =t^{2-\alpha} \int_{0}^{1}(1-\tau)^{1-\alpha} d \tau+t^{2} \int_{0}^{1} \tau^{\alpha}(1-\tau)^{1-\alpha} d \tau \\
& =\frac{1}{2-\alpha} t^{2-\alpha}+B(\alpha+1,2-\alpha) t^{2}
\end{aligned}
$$

one has

$$
\left|\int_{0}^{t}(t-s)^{1-\alpha}\left(1+s^{\alpha}\right) \frac{u_{n}(s)}{1+s^{\alpha}} d s\right| \leqslant \frac{M}{2-\alpha} t^{2-\alpha}+B(\alpha+1,2-\alpha) M t^{2} .
$$

According to the Lebesgue's dominated convergence theorem, we deduce that

$$
\begin{aligned}
\lim _{n \rightarrow+\infty} D_{0^{+}}^{\alpha-1} u_{n}(t) & =\frac{1}{\Gamma(2-\alpha)} \lim _{n \rightarrow+\infty} \frac{d}{d t} \int_{0}^{t}(t-s)^{1-\alpha}\left(1+s^{\alpha}\right) \frac{u_{n}(s)}{1+s^{\alpha}} d s \\
& =\frac{1}{\Gamma(2-\alpha)} \frac{d}{d t} \int_{0}^{t}(t-s)^{1-\alpha}\left(1+s^{\alpha}\right) \frac{u(s)}{1+s^{\alpha}} d s=D^{\alpha-1} u(t) .
\end{aligned}
$$

Thus $\left(Y,\|\cdot\|_{Y}\right)$ is a Banach space.

Before we proceed with the existence theory, we list some conditions:

(H1) $0<\beta \eta^{2 \alpha-2}<\Gamma(2 \alpha-1)$.

$(H 2)$ The function $f:[0,+\infty) \times \mathbb{R} \times \mathbb{R} \rightarrow \mathbb{R}$ is continuous such that $\int_{0}^{+\infty}|f(s, 0,0)| d s<+\infty$.

(H3) There exists nonnegative functions $\left(1+t^{\alpha}\right) g(t), h(t) \in L^{1}[0,+\infty)$ such that $|f(t, x, \bar{x})-f(t, y, \bar{y})| \leqslant g(t)|x-y|+h(t)|\bar{x}-\bar{y}|$ for all $x, y, \bar{x}, \bar{y} \in \mathbb{R}$ and $t \in[0,+\infty)$.

Lemma 3.2. Let $e(t) \in L^{1}[0,+\infty)$. Under Hypothesis $(H 1)$, the BVP

$$
\left\{\begin{array}{l}
D_{0^{+}}^{\alpha} u(t)+e(t)=0, \quad t \in(0,+\infty), \\
u(0)=0, \quad \lim _{t \rightarrow+\infty} D_{0^{+}}^{\alpha-1} u(t)=\beta I_{0^{+}}^{\alpha-1} u(\eta),
\end{array}\right.
$$

has a unique solution given by

$$
\begin{aligned}
u(t)= & -\frac{1}{\Gamma(\alpha)} \int_{0}^{t}(t-s)^{\alpha-1} e(s) d s+\frac{\Gamma(2 \alpha-1) t^{\alpha-1}}{\Gamma(\alpha)\left(\Gamma(2 \alpha-1)-\beta \eta^{2 \alpha-2}\right)} \int_{0}^{+\infty} e(s) d s \\
& -\frac{\beta t^{\alpha-1}}{\Gamma(\alpha)\left(\Gamma(2 \alpha-1)-\beta \eta^{2 \alpha-2}\right)} \int_{0}^{\eta}(\eta-s)^{2 \alpha-2} e(s) d s .
\end{aligned}
$$

Proof. By Lemma 2.6 and since $D_{0^{+}}^{\alpha} u(t)+e(t)=0$, we have

$$
u(t)=-I_{0^{+}}^{\alpha} e(t)+c_{1} t^{\alpha-1}+c_{2} t^{\alpha-2} \text { for some constants } c_{1}, c_{2} \in \mathbb{R} .
$$

So, the solution of (3.1) can be written as

$$
u(t)=-\frac{1}{\Gamma(\alpha)} \int_{0}^{t}(t-s)^{\alpha-1} e(s) d s+c_{1} t^{\alpha-1}+c_{2} t^{\alpha-2} .
$$

From $u(0)=0$, we infer that $c_{2}=0$. In addition,

$$
\begin{aligned}
D_{0^{+}}^{\alpha-1} u(t) & =-D_{0^{+}}^{\alpha-1} I_{0^{+}}^{\alpha} e(t)+c_{1} D_{0^{+}}^{\alpha-1}\left(t^{\alpha-1}\right) \\
& =-I_{0^{+}}^{1} e(t)+c_{1} \Gamma(\alpha) \\
& =-\int_{0}^{t} e(s) d s+c_{1} \Gamma(\alpha) .
\end{aligned}
$$


It follows that

$$
\lim _{t \rightarrow+\infty} D_{0^{+}}^{\alpha-1} u(t)=-\int_{0}^{+\infty} e(s) d s+c_{1} \Gamma(\alpha)
$$

Moreover

$$
\begin{aligned}
I_{0^{+}}^{\alpha-1} u(t) & =-I_{0^{+}}^{\alpha-1} I_{0^{+}}^{\alpha} e(t)+c_{1} I_{0^{+}}^{\alpha-1}\left(t^{\alpha-1}\right) \\
& =-I_{0^{+}}^{2 \alpha-1} e(t)+c_{1} \frac{\Gamma(\alpha)}{\Gamma(2 \alpha-1)} t^{2 \alpha-2} \\
& =-\frac{1}{\Gamma(2 \alpha-1)} \int_{0}^{t}(t-s)^{2 \alpha-2} e(s) d s+c_{1} \frac{\Gamma(\alpha)}{\Gamma(2 \alpha-1)} t^{2 \alpha-2} .
\end{aligned}
$$

Hence

$$
I_{0^{+}}^{\alpha-1} u(\eta)=-\frac{1}{\Gamma(2 \alpha-1)} \int_{0}^{\eta}(\eta-s)^{2 \alpha-2} e(s) d s+c_{1} \frac{\Gamma(\alpha)}{\Gamma(2 \alpha-1)} \eta^{2 \alpha-2}
$$

which together with $\lim _{t \rightarrow+\infty} D_{0^{+}}^{\alpha-1} u(t)=\beta I_{0^{+}}^{\alpha-1} u(\eta)$ yields that

$$
c_{1}=\frac{\Gamma(2 \alpha-1)}{\Gamma(\alpha)\left(\Gamma(2 \alpha-1)-\beta \eta^{2 \alpha-2}\right)} \int_{0}^{+\infty} e(s) d s-\frac{\beta}{\Gamma(\alpha)\left(\Gamma(2 \alpha-1)-\beta \eta^{2 \alpha-2}\right)} \int_{0}^{\eta}(\eta-s)^{2 \alpha-2} e(s) d s .
$$

Therefore, the unique solution of fractional boundary value problem (3.1) is

$$
\begin{aligned}
u(t)= & -\frac{1}{\Gamma(\alpha)} \int_{0}^{t}(t-s)^{\alpha-1} e(s) d s+\frac{\Gamma(2 \alpha-1) t^{\alpha-1}}{\Gamma(\alpha)\left(\Gamma(2 \alpha-1)-\beta \eta^{2 \alpha-2}\right)} \int_{0}^{+\infty} e(s) d s \\
& -\frac{\beta t^{\alpha-1}}{\Gamma(\alpha)\left(\Gamma(2 \alpha-1)-\beta \eta^{2 \alpha-2}\right)} \int_{0}^{\eta}(\eta-s)^{2 \alpha-2} e(s) d s .
\end{aligned}
$$

Lemma 3.3. Under Assumption (H1), the solution of the BVP (3.1) can also be written as

$$
u(t)=\int_{0}^{+\infty} G(t, s) e(s) d s
$$

where $G(t, s)$ is the Green's function

$$
G(t, s)=G_{1}(t, s)+G_{2}(t, s)
$$

with

$$
G_{1}(t, s)=\frac{1}{\Gamma(\alpha)} \begin{cases}t^{\alpha-1}-(t-s)^{\alpha-1}, & 0 \leqslant s \leqslant t<+\infty \\ t^{\alpha-1}, & 0 \leqslant t \leqslant s<+\infty\end{cases}
$$

and

$$
G_{2}(t, s)=\frac{\beta t^{\alpha-1}}{\Gamma(2 \alpha-1)-\beta \eta^{2 \alpha-2}} \times \frac{1}{\Gamma(\alpha)} \begin{cases}\eta^{2 \alpha-2}-(\eta-s)^{2 \alpha-2}, & 0 \leqslant s \leqslant \eta<+\infty \\ \eta^{2 \alpha-2}, & 0 \leqslant \eta \leqslant s<+\infty\end{cases}
$$


Proof. The solution of problem (3.1) can be expressed as

$$
\begin{aligned}
& u(t)=-\frac{1}{\Gamma(\alpha)} \int_{0}^{t}(t-s)^{\alpha-1} e(s) d s+\frac{1}{\Gamma(\alpha)} \int_{0}^{+\infty} t^{\alpha-1} e(s) d s \\
& -\frac{1}{\Gamma(\alpha)} \int_{0}^{+\infty} t^{\alpha-1} e(s) d s+\frac{\Gamma(2 \alpha-1) t^{\alpha-1}}{\Gamma(\alpha)\left(\Gamma(2 \alpha-1)-\beta \eta^{2 \alpha-2}\right)} \int_{0}^{+\infty} e(s) d s \\
& -\frac{\beta t^{\alpha-1}}{\Gamma(\alpha)\left(\Gamma(2 \alpha-1)-\beta \eta^{2 \alpha-2}\right)} \int_{0}^{\eta}(\eta-s)^{2 \alpha-2} e(s) d s \\
& =-\frac{1}{\Gamma(\alpha)} \int_{0}^{t}(t-s)^{\alpha-1} e(s) d s+\frac{1}{\Gamma(\alpha)} \int_{0}^{t} t^{\alpha-1} e(s) d s \\
& +\frac{1}{\Gamma(\alpha)} \int_{t}^{+\infty} t^{\alpha-1} e(s) d s-\frac{1}{\Gamma(\alpha)} \int_{0}^{+\infty} t^{\alpha-1} e(s) d s \\
& +\frac{\Gamma(2 \alpha-1) t^{\alpha-1}}{\Gamma(\alpha)\left(\Gamma(2 \alpha-1)-\beta \eta^{2 \alpha-2}\right)} \int_{0}^{+\infty} e(s) d s \\
& -\frac{\beta t^{\alpha-1}}{\Gamma(\alpha)\left(\Gamma(2 \alpha-1)-\beta \eta^{2 \alpha-2}\right)} \int_{0}^{\eta}(\eta-s)^{2 \alpha-2} e(s) d s \\
& =\frac{1}{\Gamma(\alpha)} \int_{0}^{t}\left(t^{\alpha-1}-(t-s)^{\alpha-1}\right) e(s) d s+\frac{1}{\Gamma(\alpha)} \int_{t}^{+\infty} t^{\alpha-1} e(s) d s \\
& +\frac{\beta \eta^{2 \alpha-2}}{\Gamma(\alpha)\left(\Gamma(2 \alpha-1)-\beta \eta^{2 \alpha-2}\right)} \int_{0}^{+\infty} t^{\alpha-1} e(s) d s \\
& -\frac{\beta t^{\alpha-1}}{\Gamma(\alpha)\left(\Gamma(2 \alpha-1)-\beta \eta^{2 \alpha-2}\right)} \int_{0}^{\eta}(\eta-s)^{2 \alpha-2} e(s) d s \\
& =\frac{1}{\Gamma(\alpha)} \int_{0}^{t}\left(t^{\alpha-1}-(t-s)^{\alpha-1}\right) e(s) d s+\frac{1}{\Gamma(\alpha)} \int_{t}^{+\infty} t^{\alpha-1} e(s) d s \\
& +\frac{\beta t^{\alpha-1}}{\Gamma(\alpha)\left(\Gamma(2 \alpha-1)-\beta \eta^{2 \alpha-2}\right)} \int_{0}^{\eta}\left(\eta^{2 \alpha-2}-(\eta-s)^{2 \alpha-2}\right) e(s) d s \\
& +\frac{\beta t^{\alpha-1}}{\Gamma(\alpha)\left(\Gamma(2 \alpha-1)-\beta \eta^{2 \alpha-2}\right)} \int_{\eta}^{+\infty} \eta^{2 \alpha-2} e(s) d s \\
& =\int_{0}^{+\infty} G_{1}(t, s) e(s) d s+\int_{0}^{+\infty} G_{2}(t, s) e(s) d s \text {. }
\end{aligned}
$$

Remark 3.4. From $(H 1)$ and the definition of $G_{1}(t, s)$ and $G_{2}(t, s)$, we observe that

(a) $G_{1}$ and $G_{2}$ are continuous and nonnegative functions on $[0,+\infty) \times[0,+\infty)$.

(b) $\frac{G_{1}(t, s)}{1+t^{\alpha}} \leqslant \frac{1}{\Gamma(\alpha)}$ for $(t, s) \in[0,+\infty) \times[0,+\infty)$.

(c) $\frac{G_{2}(t, s)}{1+t^{\alpha}} \leqslant \frac{\beta \eta^{2 \alpha-2}}{\Gamma(\alpha)\left(\Gamma(2 \alpha-1)-\beta \eta^{2 \alpha-2}\right)}$ for $(t, s) \in[0,+\infty) \times[0,+\infty)$.

(d) $D_{0^{+}}^{\alpha-1} G_{1}(t, s) \leqslant 1, D_{0^{+}}^{\alpha-1} G_{2}(t, s) \leqslant \frac{\beta \eta^{2 \alpha-2}}{\Gamma(2 \alpha-1)-\beta \eta^{2 \alpha-2}}, t, s>0$.

Define the operators $T_{1}, T_{2}, T$ on $Y$ by

$$
\begin{aligned}
& \left(T_{1} u\right)(t)=\int_{0}^{+\infty} G_{1}(t, s) f\left(s, u(s), D_{0^{+}}^{\alpha-1} u(s)\right) d s, \\
& \left(T_{2} u\right)(t)=\int_{0}^{+\infty} G_{2}(t, s) f\left(s, u(s), D_{0^{+}}^{\alpha-1} u(s)\right) d s, \\
& (T u)(t)=\left(T_{1} u\right)(t)+\left(T_{2} u\right)(t) .
\end{aligned}
$$


BVP (1.1) has a solution $u$ if and only if $u$ solves the operator equation $u=T u$. We shall prove the existence of a fixed point of $T$. For this, we verify that operator $T$ satisfies all conditions of Theorem 2.8. Since the Arzela-Ascoli theorem fails to work in space $Y$, we need a modified compactness criterion to prove that $T_{1}$ is compact.

Lemma 3.5. [10] Let $Z \subseteq Y$ be a bounded set. Then $Z$ is relatively compact on $Y$ if for any $u \in Z \frac{u(t)}{1+t^{\alpha}}$ and $D_{0^{+}}^{\alpha-1} u(t)$ are equicontinuous on any compact intervals of $[0,+\infty)$ and are equiconvergent at infinity.

Definition 3.6. $\frac{u(t)}{1+t^{\alpha}}$ and $D_{0^{+}}^{\alpha-1} u(t)$ are said to be equiconvergent at infinity if and only if for all $\varepsilon>0$, there exists $\delta=\delta(\varepsilon)>0$ such that

$$
\left|\frac{u\left(t_{1}\right)}{1+t_{1}^{\alpha}}-\frac{u\left(t_{2}\right)}{1+t_{2}^{\alpha}}\right|<\varepsilon \text { and }\left|D_{0^{+}}^{\alpha-1} u\left(t_{1}\right)-D_{0^{+}}^{\alpha-1} u\left(t_{2}\right)\right|<\varepsilon,
$$

for any $t_{1}, t_{2}>\delta$ and $u \in Z$.

Let $\Omega_{r}=\left\{u \in Y: \quad\|u\|_{Y}<r\right\},(r>0)$ be the open ball of radius $r$ in $Y$.

Lemma 3.7. If $(H 1)-(H 3)$ hold, then $T\left(\bar{\Omega}_{r}\right)$ is bounded set.

Proof. From (H3) we have

$$
|f(t, x, \bar{x})| \leqslant g(t)|x|+h(t)|\bar{x}|+|f(t, 0,0)|, \quad \text { for } x, \bar{x} \in \mathbb{R} \text { and } t \in[0,+\infty) .
$$

Furthermore, from Remark 3.4, we have, for any $u \in \bar{\Omega}_{r}$,

$$
\begin{aligned}
\sup _{t \geq 0}\left|\frac{(T u)(t)}{1+t^{\alpha}}\right| \leqslant & \sup _{t \geq 0}\left|\int_{0}^{+\infty} \frac{G_{1}(t, s)}{1+t^{\alpha}} f\left(s, u(s), D_{0^{+}}^{\alpha-1} u(s)\right) d s\right| \\
& +\sup _{t \geq 0}\left|\int_{0}^{+\infty} \frac{G_{2}(t, s)}{1+t^{\alpha}} f\left(s, u(s), D_{0^{+}}^{\alpha-1} u(s)\right) d s\right| \\
\leqslant & \frac{1}{\Gamma(\alpha)} \int_{0}^{+\infty}\left|f\left(s, u(s), D_{0^{+}}^{\alpha-1} u(s)\right)\right| d s \\
& +\frac{\beta \eta^{2 \alpha-2}}{\Gamma(\alpha)\left(\Gamma(2 \alpha-1)-\beta \eta^{2 \alpha-2}\right)} \int_{0}^{+\infty}\left|f\left(s, u(s), D_{0^{+}}^{\alpha-1} u(s)\right)\right| d s \\
\leqslant & \frac{\Gamma(2 \alpha-1)}{\Gamma(\alpha)\left(\Gamma(2 \alpha-1)-\beta \eta^{2 \alpha-2}\right)} \int_{0}^{+\infty}\left|f\left(s, u(s), D_{0^{+}}^{\alpha-1} u(s)\right)\right| d s \\
\leqslant & \frac{\Gamma(2 \alpha-1)}{\Gamma(\alpha)\left(\Gamma(2 \alpha-1)-\beta \eta^{2 \alpha-2}\right)}\left(r \int_{0}^{+\infty}\left(\left(1+s^{\alpha}\right) g(s)+h(s)\right) d s\right. \\
& \left.+\int_{0}^{+\infty}|f(s, 0,0)| d s\right)<+\infty
\end{aligned}
$$


and

$$
\begin{aligned}
\sup _{t \geq 0}\left|D_{0^{+}}^{\alpha-1} T u(t)\right| \leqslant & \sup _{t \geq 0} \int_{0}^{+\infty} D_{0^{+}}^{\alpha-1} G_{1}(t, s)\left|f\left(s, u(s), D_{0^{+}}^{\alpha-1} u(s)\right)\right| d s \\
& +\sup _{t \geq 0} \int_{0}^{+\infty} D_{0^{+}}^{\alpha-1} G_{2}(t, s)\left|f\left(s, u(s), D_{0^{+}}^{\alpha-1} u(s)\right)\right| d s \\
\leqslant & \int_{0}^{+\infty}\left|f\left(s, u(s), D_{0^{+}}^{\alpha-1} u(s)\right)\right| d s \\
& +\frac{\beta \eta^{2 \alpha-2}}{\Gamma(2 \alpha-1)-\beta \eta^{2 \alpha-2}} \int_{0}^{+\infty}\left|f\left(s, u(s), D_{0^{+}}^{\alpha-1} u(s)\right)\right| d s \\
\leqslant & \frac{\Gamma(2 \alpha-1)}{\Gamma(2 \alpha-1)-\beta \eta^{2 \alpha-2}}\left(r \int_{0}^{+\infty}\left(\left(1+s^{\alpha}\right) g(s)+h(s)\right) d s\right. \\
& \left.+\int_{0}^{+\infty}|f(s, 0,0)| d s\right)<+\infty .
\end{aligned}
$$

So $\|T u\|_{Y}<+\infty$ for $u \in \bar{\Omega}_{r}$. This completes the proof.

Lemma 3.8. If $(H 1)-(H 3)$ hold, then $T_{1}: \bar{\Omega}_{r} \rightarrow Y$ is completely continuous.

Proof. Step 1. The set $T_{1}\left(\bar{\Omega}_{r}\right)$ is bounded set.

By definition of operator $T_{1}$ and relation (3.2), we have that, for any $u \in \bar{\Omega}_{r}$,

$$
\begin{aligned}
\frac{\left|T_{1} u(t)\right|}{1+t^{\alpha}} & \leqslant \frac{1}{\Gamma(\alpha)} \int_{0}^{+\infty}\left|f\left(s, u(s), D_{0^{+}}^{\alpha-1} u(s)\right)\right| d s \\
& \leqslant \frac{1}{\Gamma(\alpha)} \int_{0}^{+\infty}\left(g(s)|u(s)|+h(s)\left|D_{0^{+}}^{\alpha-1} u(s)\right|+|f(s, 0,0)|\right) d s \\
& \leqslant \frac{\|u\|_{Y}}{\Gamma(\alpha)} \int_{0}^{+\infty}\left(\left(1+s^{\alpha}\right) g(s)+h(s)\right) d s+\frac{1}{\Gamma(\alpha)} \int_{0}^{+\infty}|f(s, 0,0)| d s \\
& \leqslant \frac{r}{\Gamma(\alpha)} \int_{0}^{+\infty}\left(\left(1+s^{\alpha}\right) g(s)+h(s)\right) d s+\frac{1}{\Gamma(\alpha)} \int_{0}^{+\infty}|f(s, 0,0)| d s<+\infty .
\end{aligned}
$$

Moreover

$$
\begin{aligned}
\left|D_{0^{+}}^{\alpha-1} T_{1} u(t)\right| & \leqslant \int_{0}^{+\infty}\left|f\left(s, u(s), D_{0^{+}}^{\alpha-1} u(s)\right)\right| d s \\
& \leqslant r \int_{0}^{+\infty}\left(\left(1+s^{\alpha}\right) g(s)+h(s)\right) d s+\int_{0}^{+\infty}|f(s, 0,0)| d s<+\infty .
\end{aligned}
$$

Hence $\left\|T_{1} u\right\|_{Y}<+\infty$ for $u \in \bar{\Omega}_{r}$.

Step 2. $T_{1}$ is continuous. 
For this, let $u_{n} \rightarrow u$ as $n \rightarrow+\infty$ in $\bar{\Omega}_{r}$. From Remark 3.4 and relation (3.2), we have the estimates:

$$
\begin{aligned}
\left|\frac{\left(T_{1} u_{n}\right)(t)}{1+t^{\alpha}}-\frac{\left(T_{1} u\right)(t)}{1+t^{\alpha}}\right| \leqslant & \int_{0}^{+\infty} \frac{G_{1}(t, s)}{1+t^{\alpha}}\left|f\left(s, u_{n}(s), D_{0^{+}}^{\alpha-1} u_{n}(s)\right)-f\left(s, u(s), D_{0^{+}}^{\alpha-1} u(s)\right)\right| d s \\
\leqslant & \frac{1}{\Gamma(\alpha)} \int_{0}^{+\infty}\left|f\left(s, u_{n}(s), D_{0^{+}}^{\alpha-1} u_{n}(s)\right)-f\left(s, u(s), D_{0^{+}}^{\alpha-1} u(s)\right)\right| d s \\
\leqslant & \frac{1}{\Gamma(\alpha)} \int_{0}^{+\infty}\left(\left|f\left(s, u_{n}(s), D_{0^{+}}^{\alpha-1} u_{n}(s)\right)\right|+\left|f\left(s, u(s), D_{0^{+}}^{\alpha-1} u(s)\right)\right|\right) d s \\
\leqslant & \frac{1}{\Gamma(\alpha)} \int_{0}^{+\infty}\left(g(s)\left|u_{n}(s)\right|+h(s)\left|D_{0^{+}}^{\alpha-1} u_{n}(s)\right|+|f(s, 0,0)|\right) d s \\
& +\frac{1}{\Gamma(\alpha)} \int_{0}^{+\infty}\left(g(s)|u(s)|+h(s)\left|D_{0^{+}}^{\alpha-1} u(s)\right|+|f(s, 0,0)|\right) d s \\
\leqslant & \frac{\left\|u_{n}\right\|_{Y}}{\Gamma(\alpha)} \int_{0}^{+\infty}\left(\left(1+s^{\alpha}\right) g(s)+h(s)\right) d s+\frac{1}{\Gamma(\alpha)} \int_{0}^{+\infty}|f(s, 0,0)| d s \\
& +\frac{\|u\|_{Y}}{\Gamma(\alpha)} \int_{0}^{+\infty}\left(\left(1+s^{\alpha}\right) g(s)+h(s)\right) d s+\frac{1}{\Gamma(\alpha)} \int_{0}^{+\infty}|f(s, 0,0)| d s .
\end{aligned}
$$

Moreover

$$
\left|\frac{\left(T_{1} u_{n}\right)(t)}{1+t^{\alpha}}-\frac{\left(T_{1} u\right)(t)}{1+t^{\alpha}}\right| \leqslant \frac{2}{\Gamma(\alpha)}\left(r \int_{0}^{+\infty}\left(\left(1+s^{\alpha}\right) g(s)+h(s)\right) d s+\int_{0}^{+\infty}|f(s, 0,0)| d s\right)<+\infty .
$$

Using the continuity of $f$, we obtain that

$$
\left|f\left(s, u_{n}(s), D_{0^{+}}^{\alpha-1} u_{n}(s)\right)-f\left(s, u(s), D_{0^{+}}^{\alpha-1} u(s)\right)\right| \rightarrow 0, \text { as } n \rightarrow+\infty,
$$

which implies

$$
\left\|T_{1} u_{n}-T_{1} u\right\|_{X}=\sup _{t \geq 0}\left|\frac{\left(T_{1} u_{n}\right)(t)}{1+t^{\alpha-1}}-\frac{\left(T_{1} u\right)(t)}{1+t^{\alpha-1}}\right| \rightarrow 0
$$

uniformly as $n \rightarrow+\infty$ and

$$
\begin{aligned}
\left|D_{0^{+}}^{\alpha-1} T_{1} u_{n}(t)-D_{0^{+}}^{\alpha-1} T_{1} u(t)\right| & \leqslant \int_{0}^{+\infty}\left|f\left(s, u_{n}(s), D_{0^{+}}^{\alpha-1} u_{n}(s)\right)-f\left(s, u(s), D_{0^{+}}^{\alpha-1} u(s)\right)\right| d s \\
& \leqslant 2 r \int_{0}^{+\infty}\left(\left(1+s^{\alpha}\right) g(s)+h(s)\right) d s+2 \int_{0}^{+\infty}|f(s, 0,0)| d s<+\infty .
\end{aligned}
$$

Using again the continuity of $f$, we get

$$
\sup _{t \geq 0}\left|D_{0^{+}}^{\alpha-1} T_{1} u_{n}(t)-D_{0^{+}}^{\alpha-1} T_{1} u(t)\right| \rightarrow 0, \text { uniformly as } n \rightarrow+\infty \text {. }
$$

We conclude $\left\|T_{1} u_{n}-T_{1} u\right\|_{Y} \rightarrow 0$ uniformly as $n \rightarrow+\infty$ as claimed.

Step 3. $T_{1}: \bar{\Omega}_{r} \rightarrow Y$ is relatively compact.

According to Step 1 , the set $T_{1}\left(\bar{\Omega}_{r}\right)$ is uniformly bounded. We show that functions from $\left\{\frac{T_{1} \bar{\Omega}_{r}}{1+t^{\alpha}}\right\}$ and functions from $\left\{D_{0^{+}}^{\alpha-1} T_{1} \bar{\Omega}_{r}\right\}$ are equicontinuous on any compact intervals of $[0,+\infty)$. 
Let $I \subset[0,+\infty)$ be a compact interval. Then for any $t_{1}, t_{2} \in I$ with $t_{1}<t_{2}$ and for $u \in \bar{\Omega}_{r}$, we have

$$
\begin{aligned}
\left|\frac{\left(T_{1} u\right)\left(t_{2}\right)}{1+t_{2}^{\alpha}}-\frac{\left(T_{1} u\right)\left(t_{1}\right)}{1+t_{1}^{\alpha}}\right| \leqslant & \int_{0}^{+\infty}\left|\frac{G_{1}\left(t_{2}, s\right)}{1+t_{2}^{\alpha}}-\frac{G_{1}\left(t_{1}, s\right)}{1+t_{1}^{\alpha}}\right|\left|f\left(s, u(s), D_{0^{+}}^{\alpha-1} u(s)\right)\right| d s \\
\leqslant & \int_{0}^{+\infty}\left|\frac{G_{1}\left(t_{2}, s\right)}{1+t_{2}^{\alpha}}-\frac{G_{1}\left(t_{1}, s\right)}{1+t_{2}^{\alpha}}\right|\left|f\left(s, u(s), D_{0^{+}}^{\alpha-1} u(s)\right)\right| d s \\
& +\int_{0}^{+\infty}\left|\frac{G_{1}\left(t_{1}, s\right)}{1+t_{2}^{\alpha}}-\frac{G_{1}\left(t_{1}, s\right)}{1+t_{1}^{\alpha}}\right|\left|f\left(s, u(s), D_{0^{+}}^{\alpha-1} u(s)\right)\right| d s .
\end{aligned}
$$

Moreover

$$
\begin{aligned}
& \int_{0}^{+\infty}\left|\frac{G_{1}\left(t_{2}, s\right)}{1+t_{2}^{\alpha}}-\frac{G_{1}\left(t_{1}, s\right)}{1+t_{2}^{\alpha}}\right|\left|f\left(s, u(s), D_{0^{+}}^{\alpha-1} u(s)\right)\right| d s \\
& \leqslant \quad \int_{0}^{t_{1}}\left|\frac{G_{1}\left(t_{2}, s\right)}{1+t_{2}^{\alpha}}-\frac{G_{1}\left(t_{1}, s\right)}{1+t_{2}^{\alpha}}\right|\left|f\left(s, u(s), D_{0^{+}}^{\alpha-1} u(s)\right)\right| d s \\
&+\int_{t_{1}}^{t_{2}}\left|\frac{G_{1}\left(t_{2}, s\right)}{1+t_{2}^{\alpha}}-\frac{G_{1}\left(t_{1}, s\right)}{1+t_{2}^{\alpha}}\right|\left|f\left(s, u(s), D_{0^{+}}^{\alpha-1} u(s)\right)\right| d s \\
&+\int_{t_{2}}^{+\infty}\left|\frac{G_{1}\left(t_{2}, s\right)}{1+t_{2}^{\alpha}}-\frac{G_{1}\left(t_{1}, s\right)}{1+t_{2}^{\alpha}}\right|\left|f\left(s, u(s), D_{0^{+}}^{\alpha-1} u(s)\right)\right| d s \\
& \leqslant \quad \frac{1}{\Gamma(\alpha)} \int_{0}^{t_{1}}\left|\frac{t_{2}^{\alpha-1}-t_{1}^{\alpha-1}+\left(t_{1}-s\right)^{\alpha-1}-\left(t_{2}-s\right)^{\alpha-1}}{1+t_{2}^{\alpha}}\right|\left(\left(1+s^{\alpha}\right) g(s) r+h(s) r\right. \\
&+\frac{1}{\Gamma(\alpha)} \int_{t_{1}}^{t_{2}}\left|\frac{t_{2}^{\alpha-1}-t_{1}^{\alpha-1}-\left(t_{2}-s\right)^{\alpha-1}}{1+t_{2}^{\alpha}}\right|\left(\left(1+s^{\alpha}\right) g(s) r+h(s) r+|f(s, 0,0)|\right) d s \\
&+\frac{1}{\Gamma(\alpha)} \int_{t_{2}}^{+\infty}\left|\frac{t_{2}^{\alpha-1}-t_{1}^{\alpha-1}}{1+t_{2}^{\alpha}}\right|\left(\left(1+s^{\alpha}\right) g(s) r+h(s) r+|f(s, 0,0)|\right) d s,
\end{aligned}
$$

which converges to 0 uniformly as $\left|t_{1}-t_{2}\right| \rightarrow 0$. We have also the estimates:

$$
\begin{aligned}
\int_{0}^{+\infty}\left|\frac{G_{1}\left(t_{1}, s\right)}{1+t_{2}^{\alpha}}-\frac{G_{1}\left(t_{1}, s\right)}{1+t_{1}^{\alpha}}\right|\left|f\left(s, u(s), D_{0^{+}}^{\alpha-1} u(s)\right)\right| d s \\
\quad \leqslant \int_{0}^{+\infty}\left|\frac{t_{1}^{\alpha}-t_{2}^{\alpha}}{1+t_{2}^{\alpha}}\right|\left|\frac{G_{1}\left(t_{1}, s\right)}{1+t_{1}^{\alpha}}\right|\left(\left(1+s^{\alpha}\right) g(s) r+h(s) r+|f(s, 0,0)|\right) d s \\
\leqslant \frac{1}{\Gamma(\alpha)} \int_{0}^{+\infty}\left|\frac{t_{1}^{\alpha}-t_{2}^{\alpha}}{1+t_{2}^{\alpha}}\right|\left(\left(1+s^{\alpha}\right) g(s) r+h(s) r+|f(s, 0,0)|\right) d s \rightarrow 0
\end{aligned}
$$

uniformly as $\left|t_{1}-t_{2}\right| \rightarrow 0$. Similarly, one has

$$
\begin{aligned}
\left|D_{0^{+}}^{\alpha-1} T_{1} u\left(t_{2}\right)-D_{0^{+}}^{\alpha-1} T_{1} u\left(t_{1}\right)\right| & \leqslant \int_{t_{1}}^{t_{2}}\left|f\left(s, u(s), D_{0^{+}}^{\alpha-1} u(s)\right)\right| d s \\
& \leqslant \int_{t_{1}}^{t_{2}}\left(\left(1+s^{\alpha}\right) g(s) r+h(s) r+|f(s, 0,0)|\right) d s,
\end{aligned}
$$

which converges to 0 uniformly as $\left|t_{1}-t_{2}\right| \rightarrow 0$. Then, for any $\varepsilon>0$ there exists a $\delta>0$ such that

$$
\left|\frac{\left(T_{1} u\right)\left(t_{2}\right)}{1+t_{2}^{\alpha}}-\frac{\left(T_{1} u\right)\left(t_{1}\right)}{1+t_{1}^{\alpha}}\right|<\varepsilon \quad \text { and }\left|D_{0^{+}}^{\alpha-1} T_{1} u\left(t_{2}\right)-D_{0^{+}}^{\alpha-1} T_{1} u\left(t_{1}\right)\right|<\varepsilon
$$


for all $u \in \bar{\Omega}_{r}$, as $\left|t_{2}-t_{1}\right|<\delta, t_{1}, t_{2} \in I$. This proves that the functions belong to $\left\{\frac{T_{1} \bar{\Omega}_{r}}{1+t^{\alpha}}\right\}$ and the functions belong to $\left\{D_{0^{+}}^{\alpha-1} T_{1} \bar{\Omega}_{r}\right\}$ are locally equicontinuous on $[0,+\infty)$.

Next, we show that functions from $\left\{\frac{T_{1} \bar{\Omega}_{r}}{1+t^{\alpha}}\right\}$ and functions from $\left\{D_{0^{+}}^{\alpha-1} T_{1} \bar{\Omega}_{r}\right\}$ are equiconvergent at infinity. For any $u \in \bar{\Omega}_{r}$, we have

$$
\int_{0}^{+\infty}\left|f\left(s, u(s), D_{0^{+}}^{\alpha-1} u(s)\right)\right| d s<+\infty .
$$

Considering condition (H2) and relation (3.2), for given $\varepsilon>0$, there exists a constant $L>0$ such that

$$
\int_{L}^{+\infty}\left|f\left(s, u(s), D_{0^{+}}^{\alpha-1} u(s)\right)\right| d s<\varepsilon
$$

On the other hand, since $\lim _{t \rightarrow+\infty} \frac{t^{\alpha-1}}{1+t^{\alpha}}=0$, there exists a constant $\delta_{1}>0$ such that for any $t_{1}, t_{2}>\delta_{1}$,

$$
\left|\frac{t_{i}^{\alpha-1}}{1+t_{i}^{\alpha}}\right|<\frac{\varepsilon}{2},(i=1,2) \text { and }\left|\frac{t_{2}^{\alpha-1}}{1+t_{2}^{\alpha}}-\frac{t_{1}^{\alpha-1}}{1+t_{1}^{\alpha}}\right|<\varepsilon
$$

In view of $\lim _{t \rightarrow+\infty} \frac{(t-L)^{\alpha-1}}{1+t^{\alpha}}=0$, there exists a constant $\delta_{2}>L>0$ such that, for any $t_{1}, t_{2}>\delta_{2}$ and $0 \leqslant s \leqslant L$,

$$
\left|\frac{\left(t_{i}-s\right)^{\alpha-1}}{1+t_{i}^{\alpha}}\right|<\frac{\varepsilon}{2},(i=1,2) \text { and }\left|\frac{\left(t_{2}-s\right)^{\alpha-1}}{1+t_{2}^{\alpha}}-\frac{\left(t_{1}-s\right)^{\alpha-1}}{1+t_{1}^{\alpha}}\right|<\varepsilon
$$

Select $\delta>\max \left\{\delta_{1}, \delta_{2}\right\}$. Then, for any $t_{1}, t_{2}>\delta, t_{1}<t_{2}$,

$$
\begin{aligned}
\left|\frac{\left(T_{1} u\right)\left(t_{2}\right)}{1+t_{2}^{\alpha}}-\frac{\left(T_{1} u\right)\left(t_{1}\right)}{1+t_{1}^{\alpha}}\right| \leqslant & \int_{0}^{+\infty}\left|\frac{G_{1}\left(t_{2}, s\right)}{1+t_{2}^{\alpha}}-\frac{G_{1}\left(t_{1}, s\right)}{1+t_{1}^{\alpha}}\right|\left|f\left(s, u(s), D_{0^{+}}^{\alpha-1} u(s)\right)\right| d s \\
\leqslant & \frac{1}{\Gamma(\alpha)}\left(\int_{0}^{t_{1}}\left|\frac{t_{2}^{\alpha-1}-\left(t_{2}-s\right)^{\alpha-1}}{1+t_{2}^{\alpha}}-\frac{t_{1}^{\alpha-1}-\left(t_{1}-s\right)^{\alpha-1}}{1+t_{1}^{\alpha}}\right|\right. \\
& \left|f\left(s, u(s), D_{0^{+}}^{\alpha-1} u(s)\right)\right| d s \\
& +\int_{t_{1}}^{t_{2}}\left|\frac{t_{2}^{\alpha-1}-\left(t_{2}-s\right)^{\alpha-1}}{1+t_{2}^{\alpha}}-\frac{t_{1}^{\alpha-1}}{1+t_{1}^{\alpha}}\right|\left|f\left(s, u(s), D_{0^{+}}^{\alpha-1} u(s)\right)\right| d s \\
& \left.+\int_{t_{2}}^{+\infty}\left|\frac{t_{2}^{\alpha-1}}{1+t_{2}^{\alpha}}-\frac{t_{1}^{\alpha-1}}{1+t_{1}^{\alpha}}\right|\left|f\left(s, u(s), D_{0^{+}}^{\alpha-1} u(s)\right)\right| d s\right) .
\end{aligned}
$$


Moreover

$$
\begin{aligned}
\left|\frac{\left(T_{1} u\right)\left(t_{2}\right)}{1+t_{2}^{\alpha}}-\frac{\left(T_{1} u\right)\left(t_{1}\right)}{1+t_{1}^{\alpha}}\right| \leqslant & \frac{1}{\Gamma(\alpha)}\left(\int_{0}^{L}\left|\frac{t_{2}^{\alpha-1}-\left(t_{2}-s\right)^{\alpha-1}}{1+t_{2}^{\alpha}}-\frac{t_{1}^{\alpha-1}-\left(t_{1}-s\right)^{\alpha-1}}{1+t_{1}^{\alpha}}\right|\right. \\
& \left|f\left(s, u(s), D_{0^{+}}^{\alpha-1} u(s)\right)\right| d s \\
& +\int_{L}^{+\infty}\left|\frac{t_{2}^{\alpha-1}-\left(t_{2}-s\right)^{\alpha-1}}{1+t_{2}^{\alpha}}-\frac{t_{1}^{\alpha-1}-\left(t_{1}-s\right)^{\alpha-1}}{1+t_{1}^{\alpha}}\right| \\
& \left|f\left(s, u(s), D_{0^{+}}^{\alpha-1} u(s)\right)\right| d s \\
& +\int_{L}^{+\infty}\left|\frac{t_{2}^{\alpha-1}-\left(t_{2}-s\right)^{\alpha-1}}{1+t_{2}^{\alpha}}-\frac{t_{1}^{\alpha-1}}{1+t_{1}^{\alpha}}\right|\left|f\left(s, u(s), D_{0^{+}}^{\alpha-1} u(s)\right)\right| d s \\
& +\int_{L}^{+\infty} \mid \frac{t_{2}^{\alpha-1}}{\left.1+t_{2}^{\alpha}-\frac{t_{1}^{\alpha-1}}{1+t_{1}^{\alpha}}|| f\left(s, u(s), D_{0^{+}}^{\alpha-1} u(s)\right) \mid d s\right)} \\
\leqslant & \frac{1}{\Gamma(\alpha)}\left(\sup _{s \in[0, L], u \in \bar{\Omega}_{r}}\left|f\left(s, u(s), D_{0^{+}}^{\alpha-1} u(s)\right)\right| 2 L \varepsilon+\frac{9}{2} \varepsilon^{2}\right)
\end{aligned}
$$

and

$$
\begin{aligned}
\left|D_{0^{+}}^{\alpha-1} T_{1} u\left(t_{2}\right)-D_{0^{+}}^{\alpha-1} T_{1} u\left(t_{1}\right)\right| & \leqslant \int_{t_{1}}^{t_{2}}\left|f\left(s, u(s), D_{0^{+}}^{\alpha-1} u(s)\right)\right| d s \\
& \leqslant \int_{L}^{+\infty}\left|f\left(s, u(s), D_{0^{+}}^{\alpha-1} u(s)\right)\right| d s<\varepsilon
\end{aligned}
$$

which imply that the functions from $\left\{\frac{T_{1} \bar{\Omega}_{r}}{1+t^{\alpha}}\right\}$ and the functions from $\left\{D_{0^{+}}^{\alpha-1} T_{1} \bar{\Omega}_{r}\right\}$ are equiconvergent at infinity. By Lemma 3.5, we deduce that $T_{1}: \bar{\Omega}_{r} \rightarrow Y$ is relatively compact. This ends the proof of the Lemma.

Lemma 3.9. If $(H 1)-(H 3)$ hold and

$(H 4) \frac{\beta \eta^{2 \alpha-2}}{\Gamma(2 \alpha-1)-\beta \eta^{2 \alpha-2}} \int_{0}^{+\infty}\left(\left(1+s^{\alpha}\right) g(s)+h(s)\right) d s<\Gamma(\alpha)$.

Then $T_{2}: \bar{\Omega}_{r} \rightarrow Y$ is a contractive mapping.

Proof. According to Remark 3.4 and condition (H3), we have

$$
\begin{aligned}
\left|\frac{T_{2} u(t)}{1+t^{\alpha}}-\frac{T_{2} v(t)}{1+t^{\alpha}}\right| \leqslant & \int_{0}^{+\infty} \frac{G_{2}(t, s)}{1+t^{\alpha}}\left|f\left(s, u(s), D_{0^{+}}^{\alpha-1} u(s)\right)-f\left(s, v(s), D_{0^{+}}^{\alpha-1} v(s)\right)\right| d s \\
\leqslant & \frac{\beta \eta^{2 \alpha-2}}{\Gamma(\alpha)\left(\Gamma(2 \alpha-1)-\beta \eta^{2 \alpha-2}\right)} \int_{0}^{+\infty}\left(\sup \left|\frac{u(s)-v(s)}{1+s^{\alpha}}\right|\left(1+s^{\alpha}\right) g(s)\right. \\
& \left.+\sup \left|D_{0^{+}}^{\alpha-1} u(s)-D_{0^{+}}^{\alpha-1} v(s)\right| h(s)\right) d s, \text { for all } u, v \in \bar{\Omega}_{r} \text { and } t \geqslant 0 .
\end{aligned}
$$

Hence

$$
\left|\frac{T_{2} u(t)}{1+t^{\alpha}}-\frac{T_{2} v(t)}{1+t^{\alpha}}\right| \leqslant \frac{\beta \eta^{2 \alpha-2}\|u-v\|_{Y}}{\Gamma(\alpha)\left(\Gamma(2 \alpha-1)-\beta \eta^{2 \alpha-2}\right)} \int_{0}^{+\infty}\left(\left(1+s^{\alpha}\right) g(s)+h(s)\right) d s
$$

and

$$
\left|D_{0^{+}}^{\alpha-1} T_{2} u(t)-D_{0^{+}}^{\alpha-1} T_{2} v(t)\right| \leqslant \frac{\beta \eta^{2 \alpha-2}\|u-v\|_{Y}}{\Gamma(2 \alpha-1)-\beta \eta^{2 \alpha-2}} \int_{0}^{+\infty}\left(\left(1+s^{\alpha}\right) g(s)+h(s)\right) d s .
$$


Since $\frac{1}{\Gamma(\alpha)} \geqslant 1$, one has

$$
\left\|T_{2} u-T_{2} v\right\|_{Y} \leqslant \frac{\beta \eta^{2 \alpha-2}}{\Gamma(\alpha)\left(\Gamma(2 \alpha-1)-\beta \eta^{2 \alpha-2}\right)}\left(\int_{0}^{+\infty}\left(\left(1+s^{\alpha}\right) g(s)+h(s)\right) d s\right)\|u-v\|_{Y} .
$$

From (H4), we infer that $T_{2}$ is contractive.

Theorem 3.10. Assume that $(H 1)-(H 4)$ hold and

(H5) There exists $\rho>0$ such that

$$
\frac{\rho\left(\Gamma(2 \alpha-1)-\beta \eta^{2 \alpha-2}\right)}{\Gamma(2 \alpha-1)\left(\rho \int_{0}^{+\infty}\left(\left(1+s^{\alpha}\right) g(s)+h(s)\right) d s+\int_{0}^{+\infty}|f(s, 0,0)| d s\right)}>\frac{1}{\Gamma(\alpha)}
$$

Then problem (1.1) has at least one solution.

Proof. Consider the parameterized BVP

$$
\left\{\begin{array}{l}
D_{0^{+}}^{\alpha} u(t)+\lambda f\left(t, u(t), D_{0^{+}}^{\alpha-1} u(t)\right)=0, \quad t>0 \\
u(0)=0, \quad \lim _{t \rightarrow+\infty} D_{0^{+}}^{\alpha-1} u(t)=\beta I_{0^{+}}^{\alpha-1} u(\eta)
\end{array}\right.
$$

for $\lambda \in(0,1)$. Solving problem (3.3) is equivalent to solving the fixed point of equation $u=\lambda T u$. Let

$$
\Omega_{\rho}=\left\{u \in Y: \quad\|u\|_{Y}<\rho\right\}
$$

for some positive constant $\rho$. From Lemma 3.7, the set $T\left(\bar{\Omega}_{\rho}\right)$ is bounded and by Lemma 3.8, the operator $T_{1}: \bar{\Omega}_{\rho} \rightarrow Y$ is completely continuous while Lemma 3.9 implies that the operator $T_{2}: \bar{\Omega}_{\rho} \rightarrow Y$ is contractive. So it remains to prove that $u \neq \lambda T u$ for $u \in \partial \Omega_{\rho}$ and $\lambda \in(0,1)$. Assume that there exists $u \in \partial \Omega_{\rho}$ with $u=\lambda T u$. The same way in the argument as Lemma 3.7 yields that, for $\lambda \in(0,1)$,

$$
\begin{aligned}
\sup _{t \geq 0}\left|\frac{u(t)}{1+t^{\alpha}}\right|= & \sup _{t \geq 0}\left|\frac{(\lambda T u)(t)}{1+t^{\alpha}}\right| \\
\leqslant & \sup _{t \geq 0}\left|\frac{(T u)(t)}{1+t^{\alpha}}\right| \\
\leqslant & \sup _{t \geq 0}\left|\int_{0}^{+\infty} \frac{G_{1}(t, s)}{1+t^{\alpha}} f\left(s, u(s), D_{0^{+}}^{\alpha-1} u(s)\right) d s\right| \\
& +\sup _{t \geq 0}\left|\int_{0}^{+\infty} \frac{G_{2}(t, s)}{1+t^{\alpha}} f\left(s, u(s), D_{0^{+}}^{\alpha-1} u(s)\right) d s\right| \\
\leqslant & \frac{\Gamma(2 \alpha-1)}{\Gamma(\alpha)\left(\Gamma(2 \alpha-1)-\beta \eta^{2 \alpha-2}\right)}\left(\rho \int_{0}^{+\infty}\left(\left(1+s^{\alpha}\right) g(s)+h(s)\right) d s\right. \\
& \left.+\int_{0}^{+\infty}|f(s, 0,0)| d s\right)
\end{aligned}
$$


and

$$
\begin{aligned}
\sup _{t \geq 0}\left|D_{0^{+}}^{\alpha-1} u(t)\right|= & \sup _{t \geq 0}\left|\lambda D_{0^{+}}^{\alpha-1} T u(t)\right| \\
\leqslant & \sup _{t \geq 0}\left|D_{0^{+}}^{\alpha-1} T u(t)\right| \\
\leqslant & \sup _{t \geq 0} \int_{0}^{+\infty} D_{0^{+}}^{\alpha-1} G_{1}(t, s)\left|f\left(s, u(s), D_{0^{+}}^{\alpha-1} u(s)\right)\right| d s \\
& +\sup _{t \geq 0} \int_{0}^{+\infty} D_{0^{+}}^{\alpha-1} G_{2}(t, s)\left|f\left(s, u(s), D_{0^{+}}^{\alpha-1} u(s)\right)\right| d s \\
\leqslant & \frac{\Gamma(2 \alpha-1)}{\Gamma(2 \alpha-1)-\beta \eta^{2 \alpha-2}}\left(\rho \int_{0}^{+\infty}\left(\left(1+s^{\alpha}\right) g(s)+h(s)\right) d s\right. \\
& \left.+\int_{0}^{+\infty}|f(s, 0,0)| d s\right) .
\end{aligned}
$$

Hence

$$
\|u\|_{Y} \leqslant \frac{\Gamma(2 \alpha-1)}{\Gamma(\alpha)\left(\Gamma(2 \alpha-1)-\beta \eta^{2 \alpha-2}\right)}\left(\rho \int_{0}^{+\infty}\left(\left(1+s^{\alpha}\right) g(s)+h(s)\right) d s+\int_{0}^{+\infty}|f(s, 0,0)| d s\right)
$$

and

$$
\rho \leqslant \frac{\Gamma(2 \alpha-1)}{\Gamma(\alpha)\left(\Gamma(2 \alpha-1)-\beta \eta^{2 \alpha-2}\right)}\left(\rho \int_{0}^{+\infty}\left(\left(1+s^{\alpha}\right) g(s)+h(s)\right) d s+\int_{0}^{+\infty}|f(s, 0,0)| d s\right) .
$$

This implies that

$$
\frac{\rho \Gamma(\alpha)\left(\Gamma(2 \alpha-1)-\beta \eta^{2 \alpha-2}\right)}{\Gamma(2 \alpha-1)\left(\rho \int_{0}^{+\infty}\left(\left(1+s^{\alpha}\right) g(s)+h(s)\right) d s+\int_{0}^{+\infty}|f(s, 0,0)| d s\right)} \leqslant 1,
$$

which contradicts condition (H5). With Theorem 2.8, we conclude that BVP (1.1) has at least one solution.

\section{EXAMPLES}

Example 4.1. Consider the BVP on infinite interval

$$
\left\{\begin{array}{l}
D_{0^{+}}^{\frac{3}{2}} u(t)+\frac{u(t)}{(28+t)^{2}\left(1+\sqrt{t^{3}}\right)}+\frac{D_{0^{+}}^{\frac{1}{2}} u(t)}{3 e^{t}-1}+e^{-t}=0, \quad t>0 \\
u(0)=0, \quad \lim _{t \rightarrow+\infty} D_{0^{+}}^{\frac{1}{2}} u(t)=\frac{1}{2} I_{0^{+}}^{\frac{1}{2}} u(1) .
\end{array}\right.
$$

In this case, $\alpha=\frac{3}{2}, \Gamma\left(\frac{3}{2}\right)=\frac{\sqrt{\pi}}{2}, \Gamma(2 \alpha-1)=\Gamma(2)=1, \beta=\frac{1}{2}, \eta=1$. Let

$$
f(t, x, y)=\frac{x}{(28+t)^{2}\left(1+\sqrt{t^{3}}\right)}+\frac{y}{3 e^{t}-1}+e^{-t}
$$

and

$$
g(t)=\frac{1}{(28+t)^{2}\left(1+\sqrt{t^{3}}\right)}, \quad h(t)=\frac{1}{3 e^{t}-1} .
$$

Choose

$$
\rho>\frac{1}{\frac{7 \sqrt{\pi}-1}{28}-\ln \left(\frac{3}{2}\right)} .
$$


We have

$$
\begin{aligned}
\int_{0}^{+\infty}\left(1+s^{\frac{3}{2}}\right) g(s) d s & =\frac{1}{28}<+\infty, \int_{0}^{+\infty} h(s) d s=\ln \left(\frac{3}{2}\right)<+\infty \\
\int_{0}^{+\infty}|f(s, 0,0)| d s & =\int_{0}^{+\infty} e^{-s} d s=1<+\infty
\end{aligned}
$$

Then

(H1) $0<\beta \eta^{2 \alpha-2}<\Gamma(2 \alpha-1)$.

(H2) $f:[0,+\infty) \times \mathbb{R} \times \mathbb{R} \rightarrow \mathbb{R}$ is continuous and $\int_{0}^{+\infty}|f(s, 0,0)| d s<+\infty$.

$(H 3)|f(t, x, \bar{x})-f(t, y, \bar{y})| \leqslant g(t)|x-y|+h(t)|\bar{x}-\bar{y}|$, for all $x, y, \bar{x}, \bar{y} \in \mathbb{R}$ and $t \in[0,+\infty)$.

$(H 4)$

$$
\begin{aligned}
\frac{\beta \eta^{2 \alpha-2}}{\Gamma(2 \alpha-1)-\beta \eta^{2 \alpha-2}} \int_{0}^{+\infty}\left(\left(1+s^{\alpha}\right) g(s)+h(s)\right) d s & =\frac{\frac{1}{2}}{1-\frac{1}{2}}\left(\frac{1}{28}+\ln \left(\frac{3}{2}\right)\right) \\
& <\Gamma\left(\frac{3}{2}\right) .
\end{aligned}
$$

$(H 5)$

$$
\begin{aligned}
\frac{\rho\left(\Gamma(2 \alpha-1)-\beta \eta^{2 \alpha-2}\right)}{\Gamma(2 \alpha-1)\left(\rho \int_{0}^{+\infty}\left(\left(1+s^{\alpha}\right) g(s)+h(s)\right) d s+\int_{0}^{+\infty}|f(s, 0,0)| d s\right)} & =\frac{\frac{1}{2} \rho}{\left(\frac{1}{28}+\ln \left(\frac{3}{2}\right)\right) \rho+1} \\
& =\frac{\frac{1}{2}}{\frac{1}{28}+\ln \left(\frac{3}{2}\right)+\frac{1}{\rho}} \\
& >\frac{1}{\Gamma\left(\frac{3}{2}\right)} .
\end{aligned}
$$

Hence, all conditions of Theorem 3.10 are satisfied and we deduce that BVP (4.1) has at least one solution.

Example 4.2. Consider the BVP on infinite interval

$$
\left\{\begin{array}{l}
D_{0^{+}}^{\frac{5}{4}} u(t)-\frac{|u(t)| e^{-t}}{6(1+|u(t)|)\left(1+\sqrt[4]{t^{5}}\right)}+\frac{1}{\left(1+\left|D_{0^{+}}^{\frac{1}{4}} u(t)\right|\right)(5+t)^{2}}+\frac{4}{5} e^{-t}=0, \quad t>0, \\
u(0)=0, \quad \lim _{t \rightarrow+\infty} D_{0^{+}}^{\frac{1}{4}} u(t)=\frac{3}{2} I_{0^{+}}^{4} u\left(\frac{1}{9}\right) .
\end{array}\right.
$$

In this case, $\alpha=\frac{5}{4}, \Gamma\left(\frac{5}{4}\right) \approx 0.906402, \Gamma(2 \alpha-1)=\Gamma\left(\frac{3}{2}\right)=\frac{\sqrt{\pi}}{2}, \beta=\frac{3}{2}, \eta=\frac{1}{9}$. Let

$$
\begin{gathered}
f(t, x, y)=-\frac{e^{-t}}{6\left(1+\sqrt[4]{t^{5}}\right)} \times \frac{|x|}{1+|x|}+\frac{1}{(5+t)^{2}(1+|y|)}+\frac{4}{5} e^{-t}, \\
g(t)=\frac{e^{-t}}{6\left(1+\sqrt[4]{t^{5}}\right)}, \quad h(t)=\frac{1}{(5+t)^{2}},
\end{gathered}
$$

and select

$$
\rho>\frac{30 \sqrt{\pi}}{30 \Gamma\left(\frac{5}{4}\right)(\sqrt{\pi}-1)-11 \sqrt{\pi}}
$$


Then

$$
\begin{aligned}
\int_{0}^{+\infty}\left(1+\sqrt[4]{s^{5}}\right) g(s) d s & =\frac{1}{6}<+\infty, \int_{0}^{+\infty} h(s) d s=\frac{1}{5}<+\infty \\
\int_{0}^{+\infty}|f(s, 0,0)| d s & =\int_{0}^{+\infty}\left(\frac{1}{(5+s)^{2}}+\frac{4}{5} e^{-s}\right) d s=1<+\infty
\end{aligned}
$$

Moreover

$$
\begin{aligned}
|f(t, x, \bar{x})-f(t, y, \bar{y})| \leqslant & \frac{e^{-t}}{6\left(1+\sqrt[4]{t^{5}}\right)}\left|\frac{|x|}{1+|x|}-\frac{|y|}{1+|y|}\right| \\
& +\frac{1}{(5+t)^{2}}\left|\frac{1}{1+|\bar{x}|}-\frac{1}{1+|\bar{y}|}\right| \\
\leqslant & \frac{e^{-t}}{6\left(1+\sqrt[4]{t^{5}}\right)} \times \frac{|x-y|}{(1+|x|)(1+|y|)} \\
& +\frac{1}{(5+t)^{2}} \times \frac{|\bar{x}-\bar{y}|}{(1+|\bar{x}|)(1+|\bar{y}|)} \\
\leqslant & g(t)|x-y|+h(t)|\bar{x}-\bar{y}|,
\end{aligned}
$$

for all $x, y, \bar{x}, \bar{y} \in \mathbb{R}$ and $t \in[0,+\infty)$.

Then

(H1) $0<\beta \eta^{2 \alpha-2}<\Gamma(2 \alpha-1)$.

(H2) $f:[0,+\infty) \times \mathbb{R} \times \mathbb{R} \rightarrow \mathbb{R}$ is continuous and $\int_{0}^{+\infty}|f(s, 0,0)| d s<+\infty$.

(H3) $|f(t, x, \bar{x})-f(t, y, \bar{y})| \leqslant g(t)|x-y|+h(t)|\bar{x}-\bar{y}|$ for all $x, y, \bar{x}, \bar{y} \in \mathbb{R}$ and $t \in[0,+\infty)$.

(H4)

$$
\begin{aligned}
\frac{\beta \eta^{2 \alpha-2}}{\Gamma(2 \alpha-1)-\beta \eta^{2 \alpha-2}} \int_{0}^{+\infty}\left(\left(1+s^{\alpha}\right) g(s)+h(s)\right) d s & =\frac{11}{30(\sqrt{\pi}-1)} \\
& <\Gamma\left(\frac{5}{4}\right) .
\end{aligned}
$$

$(H 5)$

$$
\begin{aligned}
\frac{\rho\left(\Gamma(2 \alpha-1)-\beta \eta^{2 \alpha-2}\right)}{\Gamma(2 \alpha-1)\left(\rho \int_{0}^{+\infty}\left(\left(1+s^{\alpha}\right) g(s)+h(s)\right) d s+\int_{0}^{+\infty}|f(s, 0,0)| d s\right)} & =\frac{(\sqrt{\pi}-1) \rho}{\sqrt{\pi}\left(\frac{11}{30} \rho+1\right)} \\
& =\frac{\sqrt{\pi}-1}{\sqrt{\pi}\left(\frac{11}{30}+\frac{1}{\rho}\right)} \\
& >\frac{1}{\Gamma\left(\frac{5}{4}\right)} .
\end{aligned}
$$

Hence, all conditions of Theorem 3.10 are satisfied, which guarantee that BVP (4.2) has at least one solution.

\section{Acknowledgement}

The author is very grateful to the reviewers for their valuable comments which improved the contents of this paper. 


\section{REFERENCES}

[1] B. Ahmad, S. K. Ntouyas, Existence results for nonlinear fractional differential equations with four-point nonlocal type integral boundary conditions, Afr. Diaspora J. Math. 11 (2011), 29-39.

[2] A. Domoshnitsky, Sturm theorems and distance between adjacent zeros for second order integro-differential equations, J. Nonlinear Var. Anal. 2 (2018), 155-164.

[3] M. Benchohra, S. Hamani, S.K. Ntouyas, Boundary value problems for differential equations with fractional order, Surv. Math. Appl. 3 (2008), 1-12.

[4] J. Caballero, J. Harjani, K. Sadarangani, Positive solutions for a class of singular fractional boundary value problems, Comput. Math. Appl. 62 (2011), 1325-1332.

[5] C. S. Goodrich, Existence of a positive solution to systems of differential equations of fractional order, Comput. Math. Appl. 62 (2011) 1251-1268.

[6] A. Guezane-Lakoud, R. Khaldi, Solvability of a fractional boundary value problem with fractional integral condition, Nonlinear Anal. 75 (2012), 2692-2700.

[7] S. Liang, J.Zhang, Existence of multiple positive solutions for m-point fractional boundary value problems of on an infinite interval, Math. Comput. Modelling 54 (2011), 1334-1346.

[8] S.K. Ntouyas, Existence results for nonlocal boundary value problems for fractional differential equations and inclusions with fractional integral boundary conditions, Discuss. Math. Differ. Incl. Control Optim. 33 (2013), 17-39.

[9] C. Shen, H. Zhou, L. Yang, On the existence of solution to a boundary value problem of fractional differential equation on the infinite interval, Boundary Value Prob. 2015 (2015), Article ID 241.

[10] X. Su, S. Zhang, Unbounded solutions to a boundary value problem of fractional order on the half-line, Comput. Math. Appl. 61 (2011), 1079-1087.

[11] Y. Wang, L. Liu, Positive solutions for fractional m-point boundary value problem in Banach spaces, Acta. Math. Sci. 32 (2012), 246-256.

[12] L. Wang, X. Zhang, Positive solutions of m-point boundary value problems for a class of nonlinear fractional differential equations, J. Appl. Math. Comput. 42 (2013), 387-399.

[13] C. Yu, J. Wang, Y. Guo, Solvability for integral boundary value problems of fractional differential equation on infinite intervals, J. Nonlinear Sci. Appl. 9 (2016), 160-170.

[14] X. Zhao, W. Ge, Unbounded solutions for a fractional boundary value problem on the infinite interval, Acta. Appl. Math. 109 (2010), 495-505.

[15] K. Oldham, J. Spanier, The fractional calculus: theory and applications of differentiation and integration to arbitrary order, 111, Elsevier, 1974

[16] A. A. Kilbas, H. M. Srivastava, J. J. Trujillo, Theory and Applications of Fractional Differential Equations, North-Holland Mathematics Studies, vol. 204. Elsevier, Amsterdam, 2006.

[17] R.P. Agarwal, M. Meehan, D. O’Regan, Fixed point theory and applications, Cambridge University Press, Cambridge, (2001). 